\title{
Ionospheric disturbance magnetic field continuation from the ground to the ionosphere using spherical elementary current systems
}

\author{
O. Amm and A. Viljanen
}

Finnish Meteorological Institute, Geophysical Research Division, P.O. Box 503, FIN - 00101 Helsinki, Finland

(Received March 1, 1999; Revised June 23, 1999; Accepted June 24, 1999)

\begin{abstract}
A new technique for continuation of the ground magnetic field caused by ionospheric currents to the ionosphere in spherical geometry is presented that makes use of elementary ionospheric current systems, which were introduced by Amm (1997) in extension of an earlier work by Fukushima (1976). The measured ground magnetic disturbance is expanded in terms of the ground magnetic effect of a spatial distribution of such elementary current systems. Using a matrix inversion technique, the scaling factors for each elementary current system, and therefrom the ionospheric equivalent currents are calculated. The technique can be applied to both global and local scales. Its advantages compared to the common field continuation techniques with Fourier (local scale), spherical cap (local to medium scale), or spherical (global scale) harmonic expansions are: 1) No fixed limitation of the spectral content has to be given for the whole analysis area, as it has to be done for the other techniques by truncation of a series expansion. 2) The locations of the elementary current systems can be chosen freely, such that they are most suitable with respect to the available measurement sites or the type of current system to be analysed. Results of the new technique are discussed in comparison to results of the spherical cap harmonic expansion method for a model of a Cowling channel.
\end{abstract}

\section{Introduction}

Being introduced as early as by Gauß (1836), continuation of the magnetic field disturbance due to external sources from the ground to the ionosphere remains a crucial step in using ground magnetometer data in ionospheric-magnetospheric research (e.g., Untiedt and Baumjohann, 1993, and references therein). While the ground magnetic field or the ground equivalent currents by themselves can only be used qualitatively for the estimation of ionospheric electrodynamic parameters, the height-continued equivalent currents at the ionospheric level can be combined with information of the ionospheric electric field via Ohm's law to spatially obtain quantitative results on ionospheric conductances, true ionospheric currents, and field-aligned currents (e.g., Richmond and Baumjohann, 1983; Inhester et al., 1992; Amm, 1998). Field continuation in general (not only from the ground to the ionosphere) is also an important tool for the construction of three-dimension geomagnetic reference models (e.g., Haines, 1985a, Torta et al., 1992, De Santis et al., 1997), or for geological applications such as to study crustal magnetic anomalies by means of satellite data (e.g., De Santis et al., 1989), or the Earth's conductivity structure (e.g., Torta and De Santis, 1996). As we shall see, the technique described in this paper can be applied to such problems as well with small modifications.

The common technique for field continuation in spherical geometry is the expansion of a magnetic potential $\Phi$ into a series of spherical harmonics, each of which is a solution

Copy right (C) The Society of Geomagnetism and Earth, Planetary and Space Sciences (SGEPSS); The Seismological Society of Japan; The Volcanological Society of Japan; The Geodetic Society of Japan; The Japanese Society for Planetary Sciences. of Laplace's equation ${ }_{\Delta} \Phi=0$ that holds in areas free of currents (e.g., Chapman and Bartels, 1940):

$$
\begin{aligned}
\Phi(r, \vartheta, \varphi)=R_{E}[ & \sum_{n=1}^{N_{i}} \sum_{m=0}^{n}\left(\frac{R_{E}}{r}\right)^{n+1} P_{n}^{m}(\cos \vartheta) \\
& \cdot\left\{g_{n}^{m, i} \cos (m \varphi)+h_{n}^{m, i} \sin (m \varphi)\right\} \\
& +\sum_{n=1}^{N_{e}} \sum_{m=0}^{n}\left(\frac{r}{R_{E}}\right)^{n} P_{n}^{m}(\cos \vartheta) \\
& \left.\cdot\left\{g_{n}^{m, e} \cos (m \varphi)+h_{n}^{m, e} \sin (m \varphi)\right\}\right]
\end{aligned}
$$

where $R_{E}$ is the Earth's radius, $n$ and $m$ the integer degree and order of the associated Legendre function $P_{n}^{m},(r, \vartheta, \varphi)$ the coordinates of a spherical coordinate system, and $g_{n}^{m}$ and $h_{n}^{m}$ the spherical harmonic coefficients to be determined from the measurements, usually by means of a least-square errors method. The first sum in (1) corresponds to the part of the magnetic potential caused by internal sources, the second one to that caused by external currents (marked with superscripts ' $i$ ' and ' $e$ ' for the coefficients, respectively). Once the harmonic coeffients are determined, the magnetic potential can be continued to any radius $R_{C}$ inside the current-free area by setting $r=R_{C}$ in (1).

While this traditional spherical harmonic analysis (SHA) is well suited for global studies, problems appear if the area of interest and of measurements is confined to a part of the Earth's surface only: The SHA coefficients will then be poorly defined, or 'virtual' data points have to be added. A way out of these problems is provided by spherical cap harmonic analysis (SCHA; Haines, 1985b). Let us assume 
that a given data set can be covered by a spherical cap with midpoint $\left(\vartheta_{p}, \varphi_{p}\right)$ (in geographical coordinates) and a halfangle $\vartheta_{0}$ of the cap. The SCHA expansion of the magnetic potential $\Phi$ in the current-free region in the spherical coordinate system with the midpoint of the cap as the northern pole is (Haines, 1985b):

$$
\begin{aligned}
\Phi(r, \vartheta, \varphi)=R_{E}[ & \sum_{k=0}^{K_{i}} \sum_{m=0}^{k}\left(\frac{R_{E}}{r}\right)^{n_{k}(m)+1} P_{n_{k}(m)}^{m}(\cos \vartheta) \\
& \cdot\left\{g_{k}^{m, j} \cos (m \varphi)+h_{k}^{m, j} \sin (m \varphi)\right\} \\
& +\sum_{k=1}^{K_{e}} \sum_{m=0}^{k}\left(\frac{r}{R_{E}}\right)^{n_{k}(m)} P_{n_{k}(m)}^{m}(\cos \vartheta) \\
& \left.\cdot\left\{g_{k}^{m, e} \cos (m \varphi)+h_{k}^{m, e} \sin (m \varphi)\right\}\right]
\end{aligned}
$$

The structure of Eq. (2) is similar to Eq. (1) of SHA, but to yield appropriate basis functions on the cap, the SHA integral degree $n$ has to be replaced by a SCHA non-integral degree $n_{k}(m)$ where $k$ is an integer index. The $n_{k}(m)$ are determined by the boundary conditions for the associated Legendre functions $P_{n_{k}(m)}^{m}(\cos \vartheta)$ at $\vartheta=\vartheta_{0}$

$$
\begin{aligned}
& \frac{d P_{n_{k}(m)}^{m}\left(\cos \vartheta_{0}\right)}{d \vartheta}=0 \text { for } k-m \text { even, } \\
& P_{n_{k}(m)}^{m}\left(\cos \vartheta_{0}\right)=0 \quad \text { for } \quad k-m \text { odd, }
\end{aligned}
$$

i.e., for a given $m$ and $\vartheta_{0}$, those Legendre functions which fulfill Eq. (2) are searched with increasing $n_{k}(m)$ and are indexed by $k$. Accordingly, a definition of the Legendre functions is needed that does not rely on an integer degree $n$ (Hobson, 1931; Haines, 1985b):

$$
\begin{aligned}
& P_{n}^{m}(\cos \vartheta) \\
& =K_{n}^{m} \cdot \sin ^{m} \vartheta \\
& \quad \cdot F\left(m-n ; n+m+1 ; 1+m ; \frac{1-\cos \vartheta}{2}\right)
\end{aligned}
$$

where $F(\alpha ; \beta ; \gamma ; x)$ is the hypergeometric function and $K_{n}^{m}$ are normalisation factors (in geophysics usually Schmidt normalisation, e.g., Chapman and Bartels, 1940). Since $n_{k}(m) \geq k$, with the same number of coefficients, SCHA uses higher degree Legendre functions than SHA, and therefore obtains a better spectral resolution. SCHA is suitable for local to medium scale studies, with scale lengths in the order of magnitude of $1000 \mathrm{~km} \times 1000 \mathrm{~km}$.

Another method for field continuation on local scales when the curvature of the Earth's surface can be neglected will briefly be mentioned: $\mathrm{Be} \tilde{\Phi}\left(k_{x}, k_{y}\right)$ the Fourier transform of a magnetic potential $\Phi(x, y)$, with $x$ and $y$ denoting cartesian coordinate axes, and $k_{x}$ and $k_{y}$ the corresponding wave numbers. Then, for external sources (as we are mainly concerned with in this paper), the Fourier transformed potential on the ground at $z=0$ relates to the Fourier transformed potential on another height $z=z_{C}$ as

$$
\tilde{\Phi}\left(k_{x}, k_{y}, z_{C}\right)=\tilde{\Phi}\left(k_{x}, k_{y}, 0\right) \cdot e^{-k_{z} z_{C}}
$$

with $k_{z}=\sqrt{k_{x}^{2}+k_{y}^{2}}$ and $z$ positive downwards (cf., e.g., Untiedt and Baumjohann, 1993).
For all of the methods discussed, a minimum wavelength that can be resolved in the analysis has to be chosen globally, i.e., for the whole analysis area. For SHA, this wavelength is $\lambda_{\min }=\left(2 \pi R_{E}\right) / n_{\max }$, for SCHA it is $\lambda_{\min }=$ $\left(2 \pi R_{E}\right) / n_{k}(m)_{\max }$, and for the Fourier transform method it becomes $\lambda_{\min }=2 \pi / k_{z, \max }$, with the subscript 'max' denotes the highest value of $n, n_{k}(m)$, or $k_{z}$ used in the analysis, respectively. As shown in the application example below, the duty to choose this resolution boundary globally can lead to problems in the field continuation procedure if the spectral content of the field to be analysed is highly varying, or if the density of the measurement sites is. If the resolution boundary is adopted to the part of the analysis area with the highest spectral content of the field or the most dense measurements, the higher spherical harmonic or Fourier coefficients might become erratic due to lack of data in the remaining parts. If it is set lower, the analysis might be unable to reproduce details of the field in the former area.

In this paper, we present a method for field continution that is not based on spectral decomposition as the ones above. It expands the measured ground magnetic field into a sum of the magnetic field effect of spherical elementary current systems (SECS) placed in the ionosphere. The centers of these elementary current systems (called 'poles' here) can be placed freely, such that their locations are most suitable with respect to the density of the measurement sites or to the type of the magnetic field disturbance to be analysed.

\section{Spherical Elementary Current Systems}

Two types of spherical elementary (sheet) current systems have been defined by Amm (1997), one $\left(\vec{J}_{d f, e l}\right)$ being divergence-free and the other $\left(\vec{J}_{c f, e l}\right)$ curl-free. Written in a spherical coordinate system $\left(r^{\prime}, \vartheta^{\prime}, \varphi^{\prime}\right)$ with unit vectors $\left(\underline{e}_{r^{\prime}}, \underline{e}_{\vartheta^{\prime}}, \underline{e}_{\varphi^{\prime}}\right)$ that has its northern pole in the center (or the 'pole') of the elementary system (compare the sketch in Fig. 1), their definition is

$$
\vec{J}_{d f, e l}\left(\vec{r}^{\prime}\right)=\frac{I_{0, d f}}{4 \pi R_{I}} \cot \left(\vartheta^{\prime} / 2\right) \underline{e}_{\varphi^{\prime}}
$$

and

$$
\vec{J}_{c f, e l}\left(\vec{r}^{\prime}\right)=\frac{I_{0, c f}}{4 \pi R_{I}} \cot \left(\vartheta^{\prime} / 2\right) \underline{e}_{\vartheta^{\prime}}
$$

where $R_{I}$ is the radius of the ionosphere, assumed to be an infinitely thin layer at $100 \mathrm{~km}$ above the Earth's surface throughout this paper, and $I_{0,\left\{\begin{array}{l}d f \\ c f\end{array}\right\}}$ are called the scaling factors of the elementary systems. The curl-free elementary system (7) is associated with a field-aligned current (i.e., a divergence of $\vec{J}_{c f, e l}$ ) of magnitude $I_{0, c f}$ at its pole, and uniform, oppositely directed FACs of magnitude $-I_{0, c f} / 4 \pi R_{I}^{2}$ on the rest of the ionosphere, so that the net FAC over the whole ionosphere is zero. This curl-free elementary system is the same that Fukushima (1976) attributed to Pedersen currents in an uniformly conducting ionosphere. However, as shown by Amm (1997), for the following it is not necessary to consider how the current was actually produced.

By using Helmholtz's theorem, Amm (1997) showed that any ionospheric current density $\vec{J}$ can be uniquely constructed by a superposition of (6) and (7), placing poles of elementary systems all over the ionosphere. In fact, the elementary systems as defined above can be used to expand any 

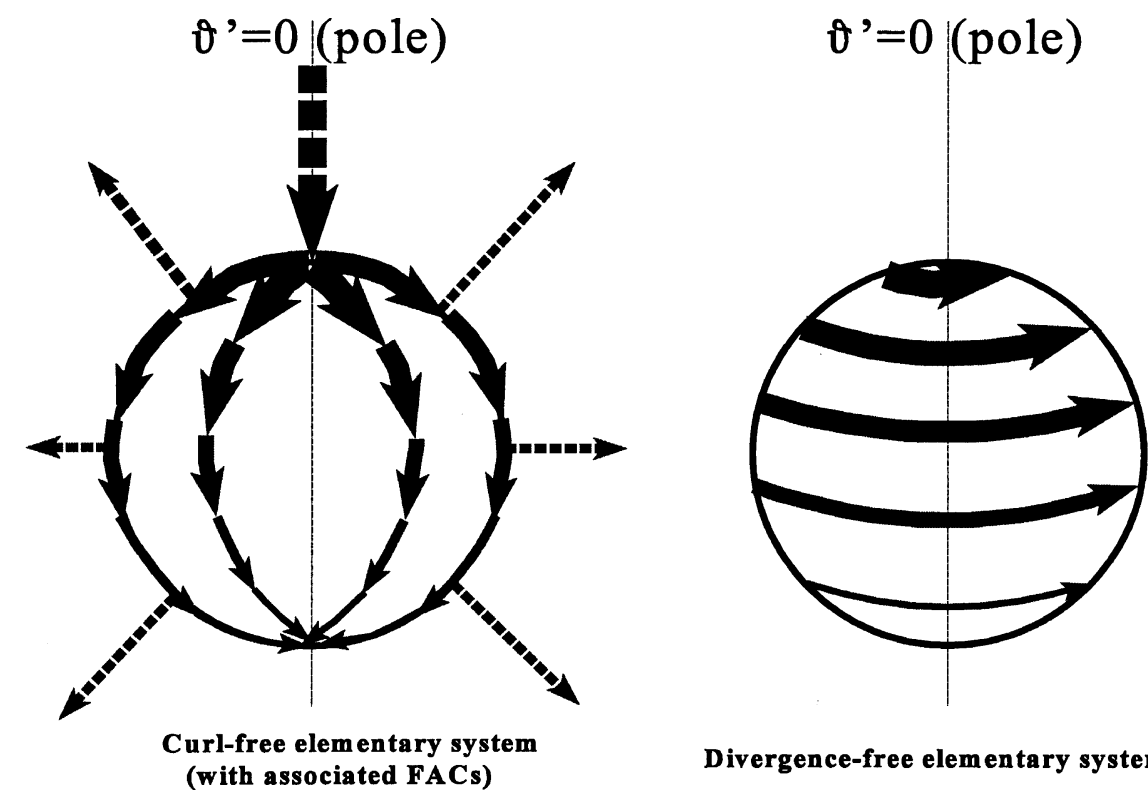

Divergence-free elementary system

Fig. 1. Sketch of spherical elementary current systems (SECS); Left side: curl-free elementary system associated with a field-aligned current (FAC) at its pole at $\vartheta^{\prime}=0$, and oppositely directed small FACs of constant magnitude on the rest of the sphere. This system does not produce any magnetic effect below the ionosphere and is not needed to expand equivalent currents; Right side: Divergence-free elementary system.

continuosly differentiable vector field on a sphere. When we are dealing with the continuation of the ground magnetic field disturbance to the ionosphere, we need to have in mind that we cannot reconstruct the total or actual current density $\vec{J}$ from it. We only obtain the equivalent currents $\vec{J}_{\text {eq,Ion }}$ which are those horizontal currents flowing in the ionosphere that produce the same magnetic effect below the ionosphere than the actual, three-dimensional current system consisting of horizontal currents and FACs (e.g., Untiedt and Baumjohann, 1993). $\vec{J}_{\text {eq,Ion }}$ is divergence-free because the curl-free part of the actual current system, like the curl-free elementary current system (7), does not produce any magnetic effect below the ionosphere, as has also been shown by Fukushima (1976).

Hence, for the purpose of our paper, i.e., the upward continuation of the ground magnetic field to the ionosphere and its representation in terms of $\vec{J}_{e q, I o n}$, we only need the divergence-free elementary system (6) to expand

$$
\vec{J}_{e q, I o n}(\vec{r})=\iint_{\text {Ionosph. }} \frac{\left[\operatorname{curl} \vec{J}\left(\vec{r}^{\prime}\right)\right]_{r}}{4 \pi R_{I}} \cot (\tilde{\vartheta} / 2) \underline{e}_{\tilde{\varphi}} d^{2} r^{\prime}
$$

where $\tilde{\vartheta}$ and $\tilde{\varphi}$ denote the coordinates of $\vec{r}$ in the spherical coordinate system with its pole at $\vec{r}^{\prime}$, and $\underline{e}_{\tilde{\vartheta}}$ and $\underline{e}_{\tilde{\varphi}}$ are the unit vectors according to this coordinate system. The $\varphi=0$ direction for each coordinate system can be defined freely, but fixed. For (8), we made use of Stokes laws to yield $\iint_{K_{r}, r \rightarrow 0}\left[\operatorname{curl} \vec{J}\left(\vec{r}^{\prime}\right)\right]_{r} d^{2} r^{\prime}=I_{0, d f}(\vec{r})$ (where $K_{r}$ is a circular ionospheric area with radius $r$ around $\vec{r}$, and $I_{0, d f}(\vec{r})$ denotes the scaling factor of the elementary current systems with pole at $\vec{r}$ ).

In case of a discrete grid representation, the integral in (8) divides into a sum over discrete (grid) points. Assuming [curl $\left.\vec{J}\left(\vec{r}^{\prime}\right)\right]_{r}=C_{G}$ to be constant over a gridpoint area $F_{G}$, the scaling factor of the elementary current system at each gridpoint $\vec{r}_{G}$ is approximated to $I_{0, d f}\left(\vec{r}_{G}\right)=C_{G} \cdot F_{G}$.

We briefly note that a similar set of elementary current systems for the planar geometry has been given in Amm (1997), equation (4). Due to a misprint in that equation, $4 \pi$ has to be replaced by $2 \pi$ in the denominator. The procedures described in this paper can be used analogously in the planar geometry by using the divergence-free one of these elementary systems instead of (6).

\section{Ground Magnetic Field Disturbance of Divergence-free Spherical Elementary Current System}

As mentioned above, the magnetic field effect of the curlfree elementary current system (7) vanishes below the ionosphere (cf. Fukushima, 1976). To calculate the ground magnetic field effect of $\vec{J}_{d f \text {,el }}$ (Eq. (6)), we derived its vector potential $\vec{A}\left(\vec{r}^{\prime}\right)$ below the ionosphere by expanding the distance between the source current filaments in the ionosphere and the point where $\vec{A}$ is evaluated in spherical harmonics. Using the generating function for the associated Legendre functions, we can write $\vec{A}$ in a closed-form expression and finally obtain $\vec{B}=\operatorname{rot} \vec{A}$. Details of this calculation are given in the appendix.

For a point with radius $r<R_{I}$ and pole angle $\vartheta^{\prime}$ from the pole of the elementary current system, the magnetic field effect of a divergence-free elementary current system $\vec{J}_{d f, e l}$ with scaling factor $I_{0}$, flowing at $r=R_{I}$, is

$$
B_{r^{\prime}}\left(r, \vartheta^{\prime}\right)=\frac{\mu_{0} I_{0}}{4 \pi r}\left(\frac{1}{\sqrt{1-\frac{2 r \cos \vartheta^{\prime}}{R_{I}}+\left(\frac{r}{R_{I}}\right)^{2}}}-1\right)
$$




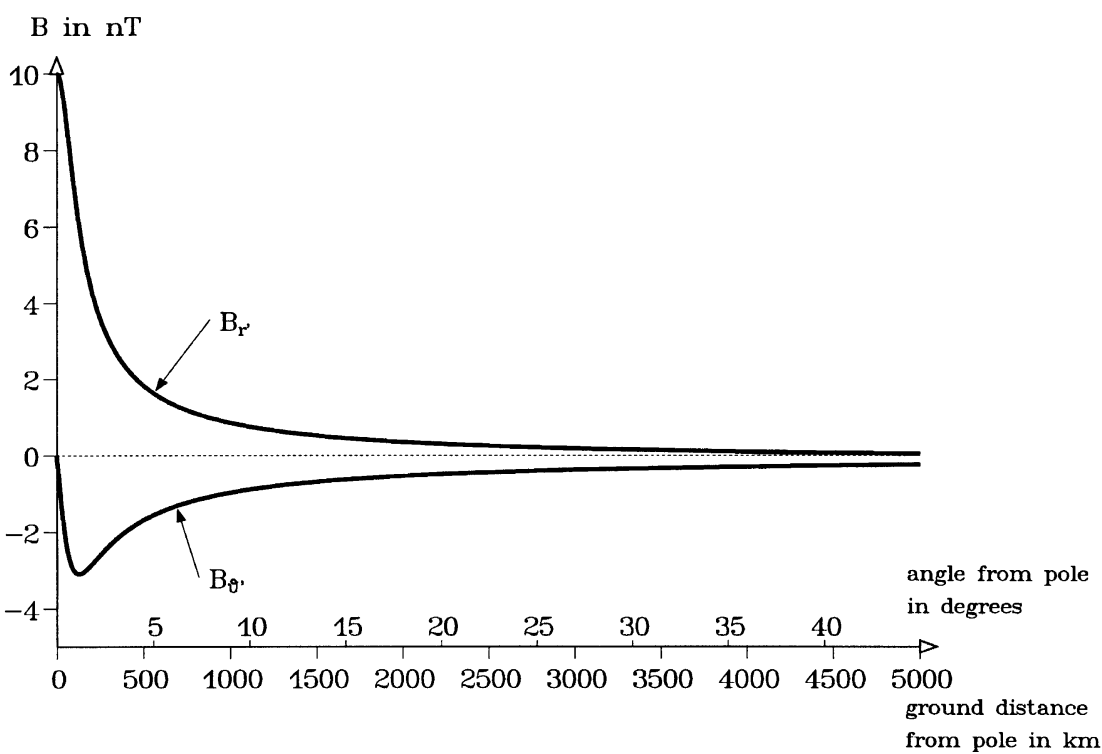

Fig. 2. Ground magnetic effect of a divergence-free elementary current system with a scaling factor $10000 \mathrm{~A}$, in the spherical coordinate system with the pole of the elementary system as the north pole, as a function of the ground distance from this pole; $B_{\varphi^{\prime}}$ is zero.

and

$$
\begin{aligned}
B_{\vartheta^{\prime}}\left(r, \vartheta^{\prime}\right)= & -\frac{\mu_{0} I_{0}}{4 \pi r \sin \vartheta^{\prime}} \\
& \cdot\left(\frac{\frac{r}{R_{I}}-\cos \vartheta^{\prime}}{\sqrt{1-\frac{2 r \cos \vartheta^{\prime}}{R_{I}}+\left(\frac{r}{R_{I}}\right)^{2}}}+\cos \vartheta^{\prime}\right)
\end{aligned}
$$

$B_{\varphi^{\prime}}$ is zero and there is no $\varphi^{\prime}$ dependence, as can be immediately seen from the symmetry. Figure 2 shows the resulting magnetic disturbances $B_{r^{\prime}}$ and $B_{\vartheta^{\prime}}$ at $r=R_{E}$, for an elementary current system with a scaling factor of $I_{0, d f}=10000 \mathrm{~A}$, as a function of the ground distance from the pole of the elementary current system. As expected, $B_{r^{\prime}}$ reaches a maximum (of exactly $10 \mathrm{nT}$ for $R_{I}-r=100 \mathrm{~km}$ ) below the pole and decreases rapidly with increasing distance from it. $B_{\vartheta^{\prime}}$ is zero directly below the pole, then shows a minimum at about $127 \mathrm{~km}$ distance from it, and decreases slowly with further increasing distance. The equations for the magnetic effect of $\vec{J}_{d f, e l}$ at $r>R_{I}$ are given in the appendix.

\section{Matrix Formulation of Expansion of Ground Magnetic Disturbance into Disturbances of Elementary Current Systems}

With the previous, the matrix formulation of the field continuation is straightforward: Let us assume that the ground magnetic field disturbance $\vec{Z}_{k}$ has been measured at points $\vec{r}_{k, o b s}=\left(\vartheta_{k, o b s}, \varphi_{k, o b s}\right), k=1, \ldots, n_{o b s}$ which are usually irregularly spaced. Poles of elementary current systems are placed at points $\vec{r}_{l, e l}=\left(\vartheta_{l, e l}, \varphi_{l, e l}\right), l=1, \ldots, n_{e l}$, which may be but do not have to be located on a regular grid. We can then determine the scaling factors of the elementary current systems that fit best to the ground magnetic field observation by solving

$$
\underline{T} \cdot \underline{I}=\underline{Z}
$$

where

$$
\underline{Z}=\left(\begin{array}{c}
Z_{1, \vartheta} \\
Z_{1, \varphi} \\
Z_{2, \vartheta} \\
Z_{2, \varphi} \\
\vdots \\
Z_{n_{o b s}, \vartheta} \\
Z_{n_{o b s}, \varphi}
\end{array}\right)
$$

is the vector of observations,

$$
\underline{I}=\left(\begin{array}{c}
I_{0, d f, 1} \\
I_{0, d f, 2} \\
\vdots \\
I_{0, d f, n_{e l}}
\end{array}\right)
$$

is the vector of the scaling factors for the elementary current systems, and

$$
\underline{\underline{T}}=\left(\begin{array}{cccc}
T_{11, \vartheta} & T_{12, \vartheta} & \cdots & T_{1 n_{e l}, \vartheta} \\
T_{11, \varphi} & T_{12, \varphi} & \cdots & T_{1 n_{e l}, \varphi} \\
T_{21, \vartheta} & T_{22, \vartheta} & \cdots & T_{2 n_{e l}, \vartheta} \\
T_{21, \varphi} & T_{22, \varphi} & \cdots & T_{2 n_{e l}, \varphi} \\
\vdots & & & \vdots \\
T_{n_{o b s} 1, \varphi} & T_{n_{o b s} 2, \varphi} & \cdots & T_{n_{o b s} n_{e l}, \varphi}
\end{array}\right) .
$$

Here, the $T_{k, l,\{}\left\{\begin{array}{l}\vartheta \\ \varphi\end{array}\right\}$ denote the $\vartheta$ or $\varphi$ component of the ground magnetic effect of an elementary current system with a scaling factor of $1 \mathrm{~A}$ and its pole at $\vec{r}_{l}$ at the observation point $\vec{r}_{k}$, expressed in the same coordinate system as the observations (usually geographic). In practice, they are easily obtained by calculating the ground magnetic effect of the elementary system using the equations as given in the Appendix, and 


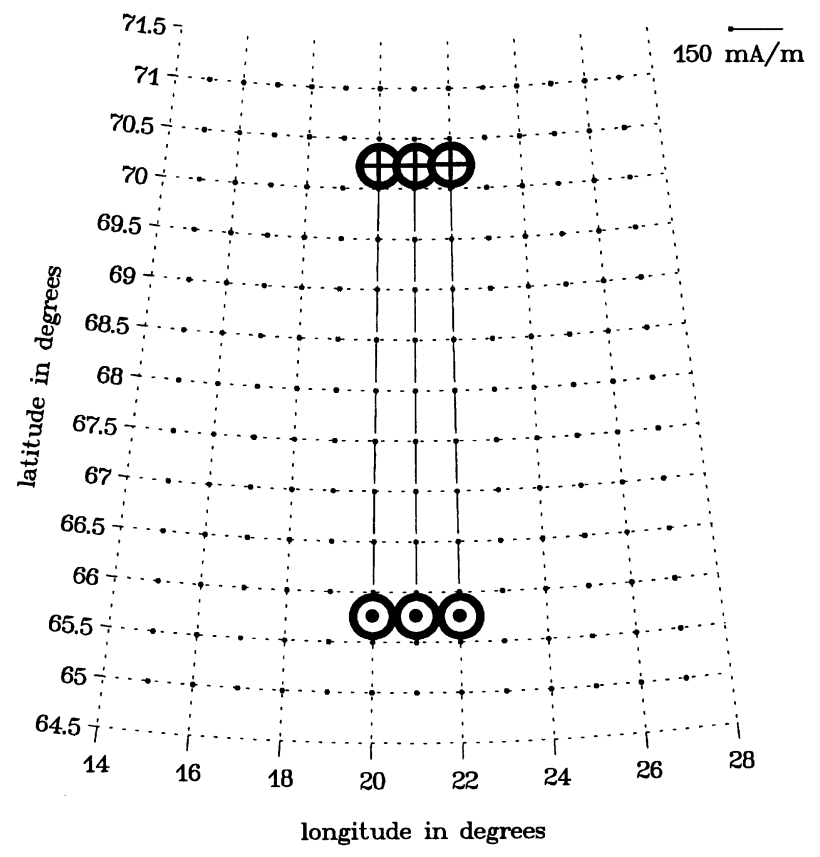

(a)

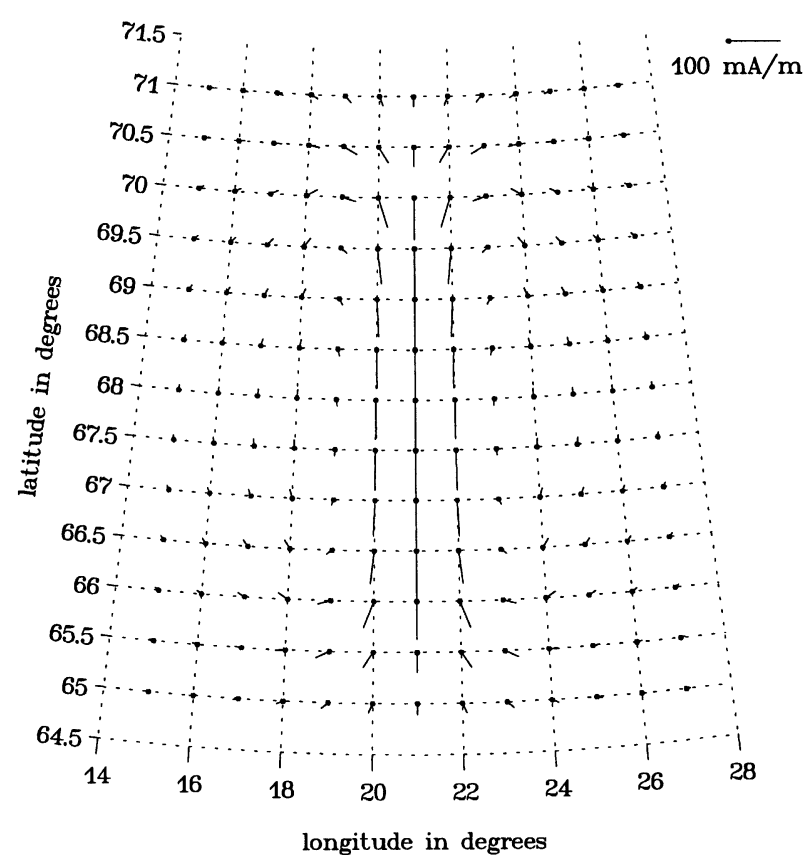

(b)

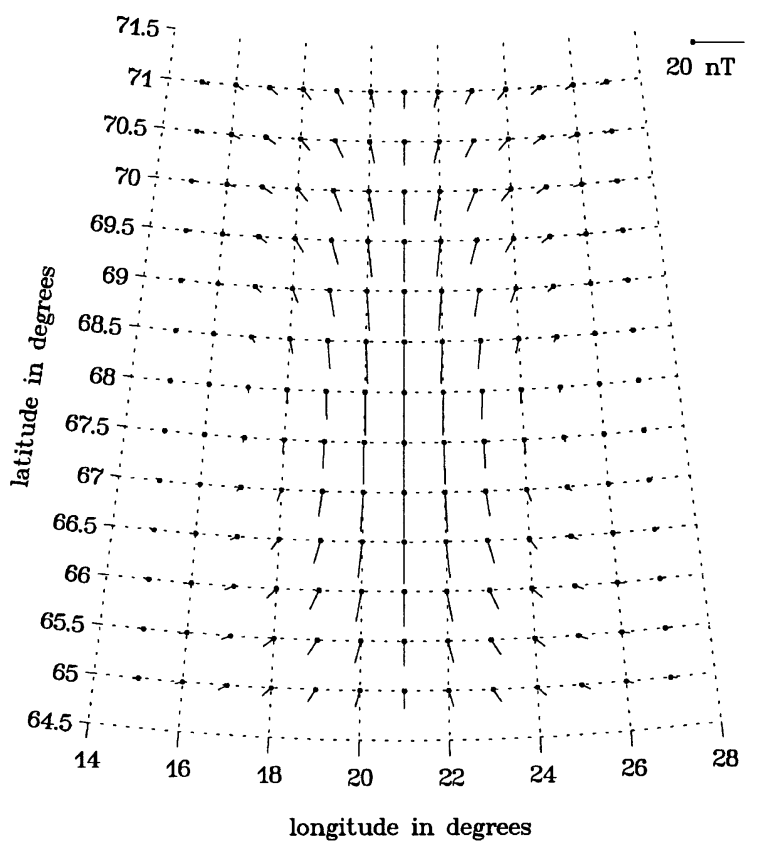

(c)

Fig. 3. Model of a Cowling channel (details see text and Amm (1997)); (a) Actual ionospheric currents and FACs (crossed circles mark downward, dotted ones upward flowing FACs).; (b) True equivalent currents; (c) By 90 degrees clockwise rotated ground magnetic disturbance.

converting it from the spherical coordinate system of the elementary system into that of the measurements.

As mentioned above, the expansion of any given equivalent current systems into a superposition of spherical elementary current systems as given in Eq. (6) is analytically unique. Since the relation between the elementary current systems and their ground magnetic effect is bijective, our expansion of the ground magnetic disturbance in terms of the elementary current system disturbances is also unique. The resulting scaling factors $\underline{I}$ are identical with those of the expansion of $\vec{J}_{\text {eq,Ion }}$ in the discrete form of Eq. (8), thus allowing to calculate $\vec{J}_{\text {eq,Ion. }}$.

\section{Matrix Equation Solving Technique}

To solve Eq. (11) for $\underline{I}$, in principle any matrix solving technique could be used. However, in practise our problem will most often be highly underdetermined, because the amount of data points $n_{o b s}$ where magnetometers have measured the ground magnetic disturbance is typically much smaller than the desired number of elementary current sys- 
tems $n_{e l}$ to obtain a good representation of $\vec{J}_{e q, I o n}$ in the ionospheric plane. In this case, $T$ will be badly conditioned, and the solution space of (11) will contain a nullspace.

A recommendable solving technique for such a case is the singular value decomposition method (SVD, e.g., Press et al., 1992): The matrix $\underline{\underline{T}}$ is decomposed into

$$
\underline{\underline{T}}=\underline{\underline{U}} \underline{\underline{w}} \underline{\underline{V^{T}}}
$$

where $\underline{\underline{U}}$ and $\underline{\underline{V}}^{T}$ are orthogonal matrices, i.e. the columns of $\underline{U}$ and the rows of $\underline{V}^{T}$ are bases for the $n_{e l}$-dimensional space $\overline{\overline{\text { of }}}$ all possible combinations of the $n_{e l}$ elementary systems. $\underline{\underline{w}}$ is diagonal, and its diagonal elements $w_{m m}, m=1, \ldots, n_{e l}$, are called the 'singular values' of $\underline{\underline{T}}$. If $n_{e l}>n_{o b s}$, those $n_{e l}-n_{o b s}$ singular values that are connected with the basis of the nullspace of $\underline{T}$ in $\underline{U}$ and $\underline{V}^{T}$ are zero. The decisive point in SVD is now that it allows to separate out other badly conditioned parts of $T$ as well, since they are connected with nonzero, but small singular values. This is done by setting all $w_{m m}$ with $w_{m m} \leq \varepsilon \cdot \operatorname{Max} .\left\{w_{m m}\right\}$ to zero. Typical values for $\varepsilon$ range between 0.01 and 0.1 . After that, (11) can be solved by

$$
\underline{I}=\underline{\underline{V}}\left(\operatorname{diag}\left(\tilde{w}_{m m}\right)\right) \underline{\underline{U}}^{T} \underline{Z}
$$

where

$$
\tilde{w}_{m m}=\left\{\begin{array}{lll}
w_{m m}^{-1} & \text { for } & w_{m m} \neq 0 \\
0 & \text { for } & w_{m m}=0
\end{array}\right.
$$

For an underdetermined system of equations, this procedure will pick the solution with minimum $|\underline{I}|^{2}$ from the total solution space (Press et al., 1992). With the solution for $I$, the ionospheric equivalent currents $\vec{J}_{\text {eq, Ion }}$ can be obtained at any ionospheric point using (8) and the remarks for the discrete formulation after that equation.

The larger $\varepsilon$ is chosen, the smoother the solution for $\vec{J}_{\text {eq, Ion }}$ will be in general. However, it should be noted that the choice of $\varepsilon$ does not invoke a spectral resolution boundary, but a separation with respect to well and badly conditioned parts of our linear system of equations.

\section{Application Example}

Finally, we test the SECS method of upward field continuation from the ground to the ionosphere in a model example of a Cowling channel and compare its results to those of the spherical cap harmonic analysis (SCHA). We choose SCHA for the comparison since on the scales of typically $1000 \mathrm{~km} \times 1000 \mathrm{~km}$ that are most interesting and applicable for upward continuation in ionospheric-magnetospheric physics (e.g., Richmond and Baumjohann, 1983; Walker et al., 1997; Amm, 1998), it is probably the most advanced existing technique (compare, e.g., Haines, 1990).

A Cowling channel is a confined ionospheric area with an enhanced conductance, leading to horizontal ionospheric currents with magnitudes that are enhanced compared to the background. The channel usually has a larger extent in the direction of the current flow (called 'channel direction') than perpendicular to it. At the edges of the Cowling channel in the channel direction, field-aligned currents (FACs) are present to feed or diverge the enhanced horizontal currents, respectively (e.g., Boström, 1974, Baumjohann et al., 1981). We will not discuss the details of this model here, since we

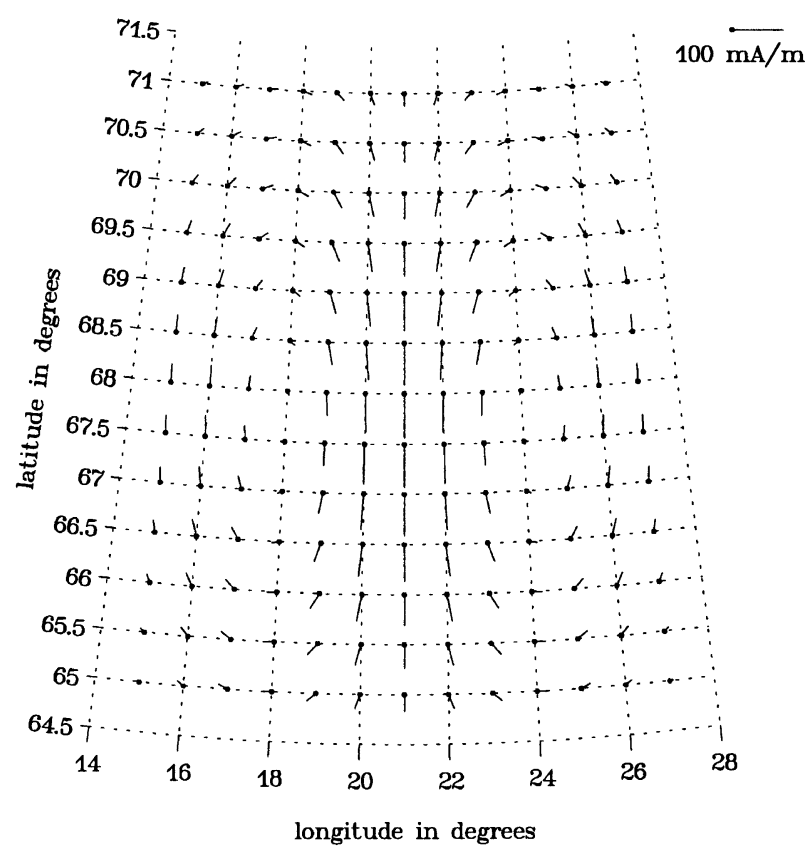

(a)

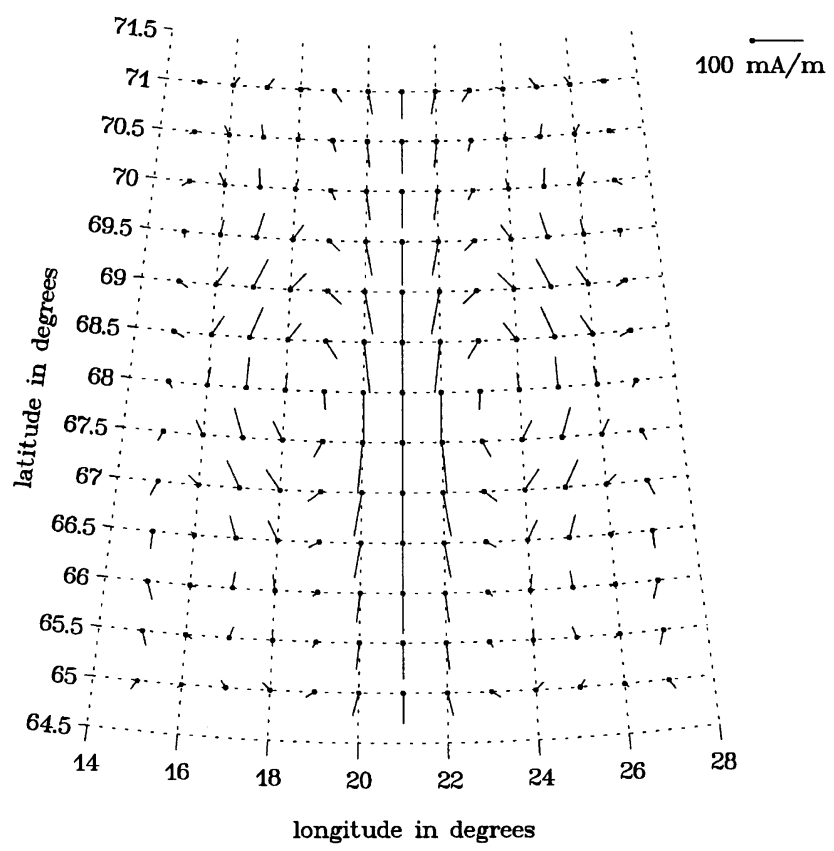

(b)

Fig. 4. Spherical cap harmonic analysis (SCHA) results of upward continuation; (a) With $K_{e}=10$; (b) Increasing to $K_{e}=12$ leads to erratic vectors.

use the same model that has been described in detail by Amm (1997), and since for the purpose of this paper only the total current flow as sketched in Fig. 3(a) is relevant. In our model, the channel is limited to the area between 66 and 70 degrees of latitude and 20 to 22 degrees of longitude. The channel currents are flowing southward, being fed at the northern and diverged at the southern end of the channel via FACs. Note that the longitude on which we place our model has no special physical meaning. It is merely for convenience adjusted to 


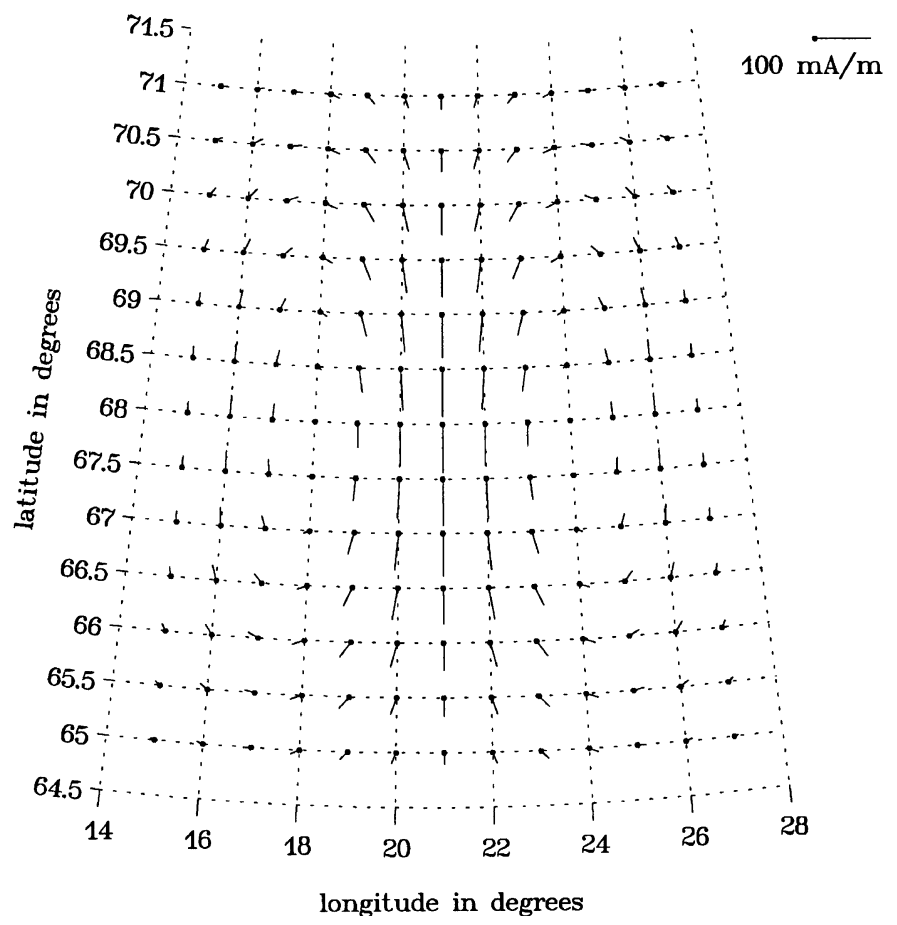

(a)

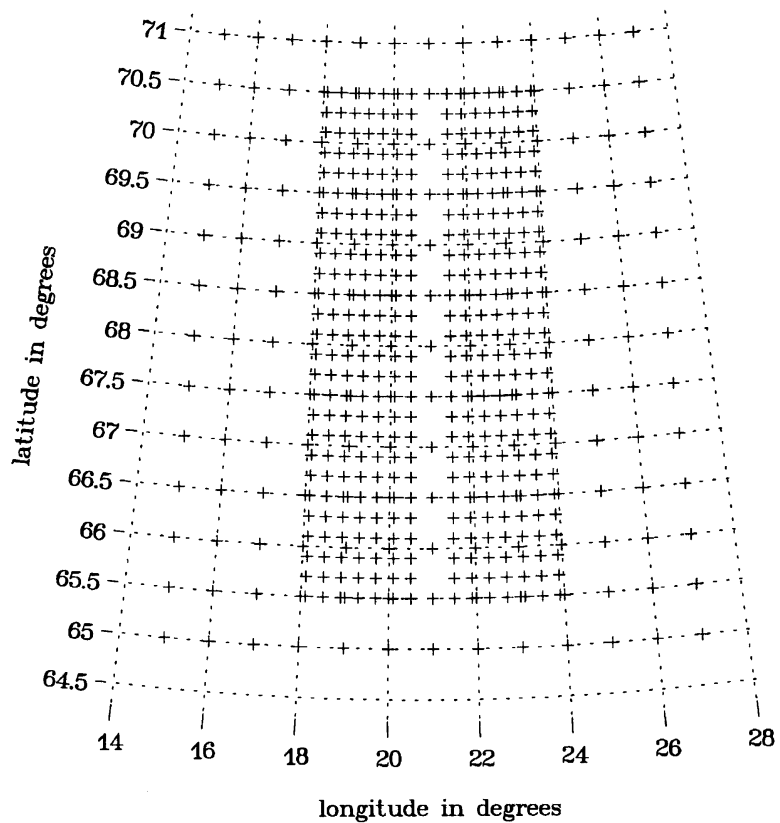

(b)

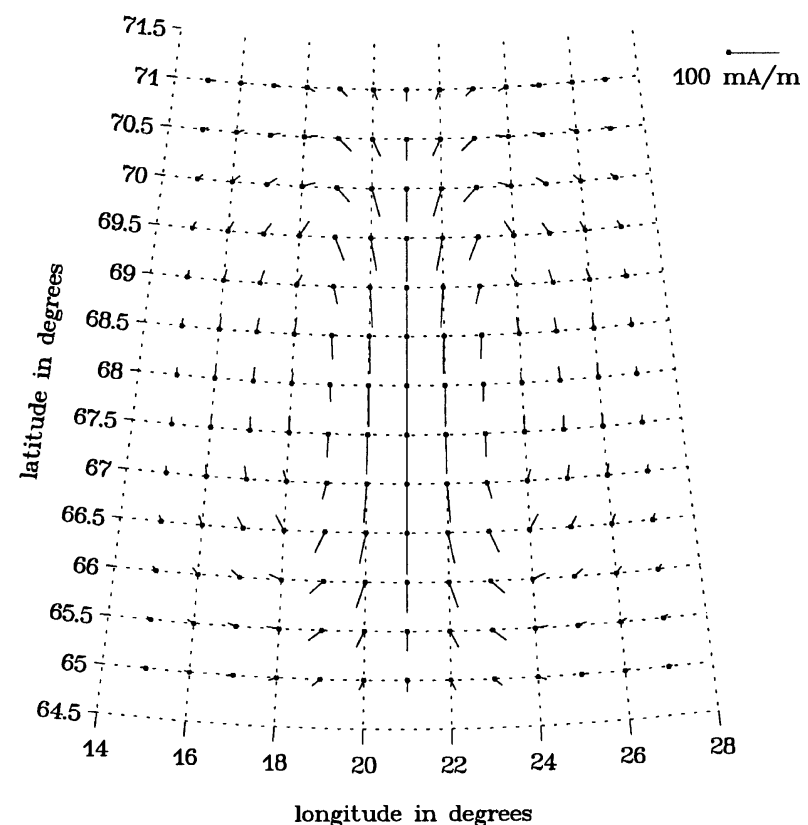

(c)

Fig. 5. Results of the spherical elementary current system (SECS) method; (a) with poles of elementary systems on the grid points shown only; (b) Refined grid in the area of loops of the by 90 degrees rotated ground magnetic field; (c) Result with poles of elementary systems on the refined grid.

\section{Northern Fennoscandia.}

The ionospheric equivalent currents $\vec{J}_{e q, \text { Ion }}$ that cause the same magnetic effect below the ionosphere as the threedimensional currents of Fig. 3(a) are shown in Fig. 3(b). As can be seen, besides the dominating southward currents in the channel area, the effect of the FACs results into two equivalent current loops at both flanks of the Cowling channel perpendicular to the channel direction. The same structure, somewhat smoothed out due to the distance from the source, can still be seen in the by 90 degrees clockwise rotated ground magnetic disturbance as calculated with Biot-Savart's law from our model currents (Fig. 3(c)).

This ground magnetic disturbance is taken as the input for the field continuation methods, and the SECS method as well as SCHA will be tested in how well they can reproduce the model equivalent current pattern as presented in Fig. 3(b). 


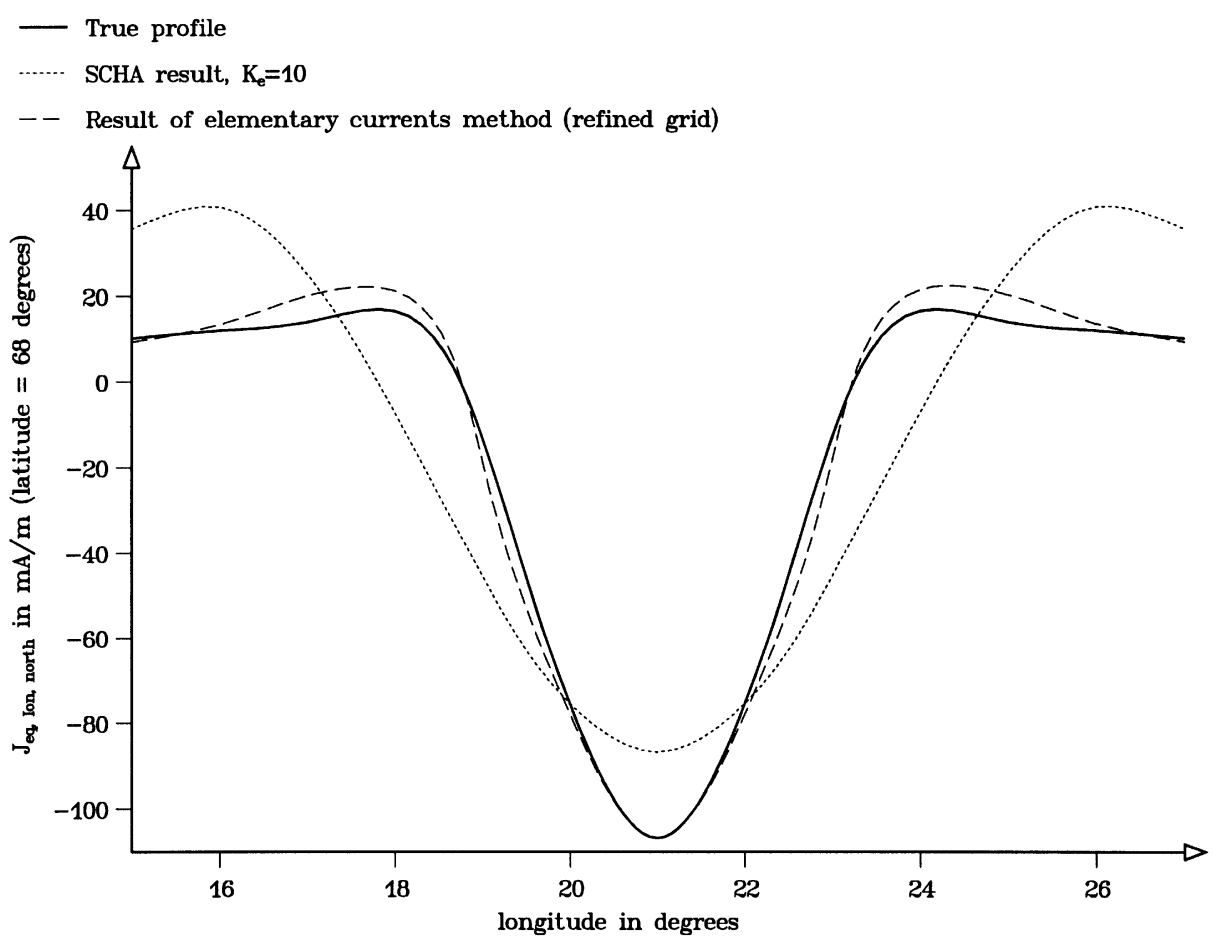

Fig. 6. Longitudinal profile of the north component of the ionospheric equivalent currents; Solid line: true profile (see Fig. 3(b)); Dotted line: SCHA upward continuation result with $K_{e}=10$ (see Fig. 4(a)); Broken line: SECS method upward continuation result with poles of elementary systems on refined grid (see Fig. 5(c)).

The SCHA algorithm used is the one of Haines (1988), with slight modifications by the authors. The equations for the equivalent currents in terms of the SCHA expansion can be found in Haines and Torta (1994).

In Fig. 4(a), the SCHA result is shown for a spherical cap half angle of $\vartheta_{0}=12^{\circ}$, and $K_{e}=10 \mathrm{in} \mathrm{Eq.} \mathrm{(2)} \mathrm{(internal} \mathrm{coef-}$ ficients are irrelevant in our model case). Since the SCHA fit is subject to errors at the boundary of the spherical cap which may continue inwards during the upward continuation, it is necessary to select a somewhat larger $\vartheta_{0}$ than would be required solely by the area of data coverage (e.g., Torta and De Santis, 1996). However, increasing $\vartheta_{0}$ to larger values than the one selected here does not anymore improve the results. Clearly, SCHA is able to reproduce the general pattern of the true ionospheric equivalent currents well. However, if the result is examined in more detail, it turns out that the currents in the center of the channel are underestimated by about $20 \mathrm{~mA} / \mathrm{m}$ (i.e., by about $20 \%$ ), whereas the equivalent return flow at the flanks perpendicular to the channel direction is larger than in the model (compare Fig. 3(b)). The smoothing of the real $\vec{J}_{e q, \text { Ion }}$ distribution that is present in the SCHA result is most obvious from the comparison of the north component of $\vec{J}_{e q, \text { Ion }}$ on a longitudinal profile at 68 degrees of latitude (Fig. 6): The SCHA result (dotted line) is not able to follow the relatively narrow peak of the model ('true') currents (solid line) in the center of the channel. The natural remedy of this is to increase $K_{e}$ for the SCHA expansion. As the result with $K_{e}=12$ shows (Fig. 4(b)), this leads to a better representation of the currents in the center of the channel, but now a population of vectors at the flanks be- come erratic (the same happens with $K_{e}=11$, but we show the $K_{e}=12$ case for clearer illustration). The same effect is observed if $\vartheta_{0}$ is decreased. The reason for this behaviour is that the spectral components that are needed to represent the peak in the center of the channel, are ill-determined at its flanks and lead to a 'swinging' effect there.

The results of the SECS method is shown in Fig. 5. The value of $\varepsilon$ used is 0.015 which was obtained in an optimisation process as the $K_{e}$ for SCHA. First, we allow poles of spherical elementary systems on the grid points of our model only. The upward continued $\vec{J}_{\text {eq, Ion }}$ essentially shows the same problems as dicussed above for SCHA in this case, as can be seen in Fig. 5(a). However, from the geometry of the ground magnetic disturbance (see Fig. 3(c)), it is obvious that the curl of the equivalent current system - and therefore also the magnitude of the scaling factors of our elementary current systems-peaks near the flanks of the channel perpendicular to the channel direction. Hence, in these areas we refine the grid for the poles of the elementary current systems as shown in Fig. 5(b). While no general "optimum rule" for such a refinement exists, from our experience a good choice is to decrease the grid spacing for the poles of the elementary current systems in those areas to about one third of the average spacing of the input data, i.e., here of the grid spacing of our model. A refinement in areas where the curl of the measured equivalent currents is small will not improve the results. The result of the SECS method using the refined grid is shown in Fig. 5(c). Although the improvement is not easily visible on the first view from this vector plot, it becomes obvious by a comparison of the $\vec{J}_{\text {eq,Ion, north }}$ 
profiles at 68 degrees of latitude (Fig. 6). The solution based on elementary current (broken line) systems follows the true profile much closer than the SCHA result (dotted line). Especially, it reproduces the minimum in the center of the channel nearly exactly, and follows the narrow shape of the true profile there closely. Such on the first view small differences may become important if the upward continued equivalent currents are used quantitatively in combination with, e.g., the ionospheric electric field to determine other ionospheric electrodynamic parameters (e.g., Inhester et al., 1992; Amm, 1998).

This application example shows that the SECS method is able to obtain at least as good, and with an appropriate grid refinement even better results in upward continuation than the already quite advanced SCHA method. In particular, a refinement of the grid does not lead to numerical problems as does the increase of spectral content by increasing $K_{e}$ in SCHA. Additionally, the SECS method can even provide some information of equivalent current sources outside the area of measurements by placing poles of elementary currents there and fitting their magnetic effect on the measurements.

\section{Conclusions and Outlook}

We have introduced a new method for field continuation, worked out in this paper for the purpose of upward continuation of the ground magnetic field disturbance to the ionosphere, and its representation as ionospheric equivalent currents. In contrast to existing methods that rely on a spectral decomposition of the magnetic potential, this method is based on spherical elementary current systems (SECS) as introduced in Amm (1997). Thus, no fixed boundary for the minimum wavelength to be resolved has to be given over the total analysis area like in the existing methods by terminating a series, but poles of elementary systems can freely be placed as they are most suitable with respect to the density of mesurements or the type of disturbance to be analysed. The value of this advantage was demonstrated in the model example of a Cowling channel where the new method, with appropriately placed elementary systems, gained a better reconstruction of the true ionospheric equivalent currents than the spherical cap harmonic analysis (SCHA). We have selected the Cowling channel model with its relatively simple geometry for this paper in order to illustrate the effects of the different upward continuation methods most clearly. The authors have tested the SECS method also for current systems of more complex geometry, and in all cases gained upward continuation results of similar quality as shown in this paper.

The method presented in this paper can easily be adapted to other field continuation problems than from the ground to the ionosphere, just by placing similar spherical elementary currents like in (6) on the sphere(s) where the currents that cause the field are assumed to flow and calculating the magnetic field effect of the elementary current system with scaling factor of $1 \mathrm{~A}$ on the sphere(s) where the measurements were taken (of course, $R_{I}$ in Eq. (6) has to be replaced by the respective radius of the sphere(s)). Likewise, separation of the parts of a field caused by external and internal sources can be carried out with the SECS method by placing elementary current systems on two spheres, one representing the external, the other the internal part. Typically, the outer shell would be the ionosphere, whereas the inner one might be placed with respect to a 'perfect conductor' that is used to approximately replace the real conductivity stucture of the Earth (e.g., Baumjohann et al., 1981; Gustafsson et al., 1981, Viljanen et al., 1995). It should, however, be noted that the introduction of more elementary current systems for a given amount of data will increase the underdetermination of the system of linear equations in (11). Furthermore, to perform the field separation, measurements of $B_{r}$ and corresponding transfer functions have to be included into Eqs. (12) and (14), respectively.

Finally, all of the upward continuation methods mentioned in this paper can also be used to derive models of the measured quantities, and thereby to inter- or extrapolate $\vec{J}_{e q, I o n}$, or the measured ground magnetic field disturbance, since the determination of the SECS, SCHA, SHA, or Fourier coefficients allows in principle to calculate these quantities at any place in the respective shells. Not surprisingly, the quality of the result will depend on the data coverage near the point where the quantities are to be interpolated. Also for this purpose, the SECS method has shown to be more robust than the spectral methods.

Acknowledgments. The authors like to thank K. H. Glaßmeier (Braunschweig) for valuable discussions, and P. Janhunen (Helsinki) for his comments on the manuscript. The work of $\mathrm{O}$. A. was supported by a DAAD-fellowship HSP III, financed by the German Ministery for Research and Technology. The Editor thanks J. M. Torta and another referee for their assistance in evaluating this paper.

\section{Appendix}

In this appendix, we outline the calculation of the magnetic field disturbance of a divergence-free elementary current system $\vec{J}_{d f, e l}$ flowing in the ionosphere at radius $R_{I}$. All calculations are done in the coordinate system with the pole of the elementary system at $\vartheta^{\prime}=0$, i.e., at the north pole. For simplicity, we omit the quotes used in the main text to separate this coordinate system from the geographical one here. We first show the derivation of Eqs. (9) and (10) for $r<R_{I}$, and then give similar equations for $r>R_{I}$.

First we insert Eq. (6) into the expression of the vector potential $\vec{A}$ of the desired magnetic field disturbance to obtain

$$
\vec{A}(\vec{r})=\frac{\mu_{0} I_{0}}{16 \pi^{2} R_{I}} \int d^{3} r^{\prime} \delta\left(r^{\prime}-R_{I}\right) \frac{\cot \vartheta^{\prime} / 2}{\left|\vec{r}-\vec{r}^{\prime}\right|} \underline{e}_{\varphi^{\prime}} .
$$

By expanding line $\left|\vec{r}-\vec{r}^{\prime}\right|^{-1}$ into spherical harmonics and using their orthogonality relations (e.g., Arfken, 1985) we arrive at

$$
\vec{A}(r, \vartheta)=\frac{\mu_{0} I_{0}}{4 \pi} \sum_{n=1}^{\infty} \frac{1}{n(n+1)}\left(\frac{r}{R_{I}}\right)^{n} P_{n}^{1}(\cos \vartheta) \underline{e}_{\varphi} .
$$

With the definitions $\lambda:=r / R_{I} x:=\cos \vartheta$, and

$$
f(\lambda):=\sum_{n=1}^{\infty} \frac{1}{n(n+1)} \lambda^{n+1} P_{n}^{1}(x)
$$

and consequently

$$
f^{\prime \prime}(\lambda)=\frac{1}{\lambda} \sum_{n=1}^{\infty} \lambda^{n} P_{n}^{1}(x)
$$


we can use the generating function for the associated Legendre polynomials $P_{n}^{m}(x)$ for the special case $m=1$ (e.g., Arfken, 1985)

$$
\begin{aligned}
\sum_{n=0}^{\infty} \lambda^{n} P_{n+1}^{1}(x) & =\frac{1}{\lambda} \sum_{n=1}^{\infty} \lambda^{n} P_{n}^{1}(x) \\
& =\frac{\sqrt{1-x^{2}}}{\left(1-2 x \lambda+\lambda^{2}\right)^{3 / 2}}
\end{aligned}
$$

to obtain a closed-form expression for $\vec{A}$ after solving the differential equation for $f(\lambda)$ :

$$
\begin{aligned}
\vec{A}(r, \vartheta)= & \frac{\mu_{0} I_{0}}{4 \pi} \frac{R_{I}}{r \sin \vartheta} \\
& \cdot\left(\sqrt{1-\frac{2 r \cos \vartheta}{R_{I}}+\left(\frac{r}{R_{I}}\right)^{2}}+\frac{r \cos \vartheta}{R_{I}}-1\right) \underline{e}_{\varphi}
\end{aligned}
$$

From (A.6), we can directly derive (9) and (10) by using $\vec{B}=\operatorname{rot} \vec{A}$.

The equations for the magnetic effect of $\vec{J}_{d f, \text { el }}$ for $r>R_{I}$ can be derived analogously to the above. The results are

$$
B_{r}(r, \vartheta)=\frac{\mu_{0} I_{0}}{4 \pi} \frac{R_{I}}{r^{2}}\left(\frac{1}{\sqrt{1-\frac{2 R_{I} \cos \vartheta}{r}+\left(\frac{R_{I}}{r}\right)^{2}}}-1\right)
$$

and

$$
\begin{aligned}
B_{\vartheta}(r, \vartheta)= & -\frac{\mu_{0} I_{0}}{4 \pi} \frac{1}{r \sin \vartheta} \\
& \cdot\left(\frac{r-R_{I} \cos \vartheta}{\sqrt{r^{2}-2 r R_{I} \cos \vartheta+R_{I}^{2}}}-1\right) .
\end{aligned}
$$

Of course, the ionospheric radius $R_{I}$ can be replaced in (A.7) and (A.8) as well as in (9) and (10) by any other radius where the elementary current system is assumed to flow.

\section{References}

Amm, O., Ionospheric elementary current systems in spherical coordinates and their application, J. Geomag. Geoelectr., 49, 947-955, 1997.

Amm, O., Method of characteristics in spherical geometry applied to a Harang discontinuity situation, Ann. Geophys., 16, 413-424, 1998.

Arfken, G., Mathematical Methods for Physicists, Academic Press, pp. 985, San Diego, U.S.A., 1985.

Baumjohann, W., R. J. Pellinen, H. J. Opgenoorth, and E. Nielsen, Joint twodimensional observations of ground magnetic and ionospheric electric fields associated with auroral zone currents: Current system associated with local auroral break-ups, Planet. Space Sci., 29, 431-447, 1981.
Boström, R., Ionosphere-magnetosphere coupling, in Magnetospheric Physics, edited by B. M. McCormack, p. 45, D. Reidel, Norwell, Mass., 1974.

Chapman, S. and J. Bartels, Geomagnetism, vol. II, pp. 1049, Oxford University Press, New York, 1940.

De Santis, A., D. J. Kerridge, and D. R. Barraclough, A spherical cap harmonic model of the crustal magnetic anomaly field in Europe observed by MAGSAT, in Geomagnetism and Palaeomagnetism, edited by F. J. Lowes et al., pp. 1-17, 1989.

De Santis, A., C. Falcone, and J. M. Torta, SHA vs. SCHA for modelling secular variation in a small region such as Italy, J. Geomag. Geoelectr., 49, 359-371, 1997.

Fukushima, N., Generalized theorem for no ground magnetic effect of vertical currents connected with Pedersen currents in the uniform-conductivity ionosphere, Rep. Ionos. Space Res. Japan., 30, 35-40, 1976.

Gauß, C. F., Erdmagnetismus und Erdmagnetometer, in: Gauß, C. F., Werke, hrsg. von der Königlichen Gesellschaft der Wissenschaften, Göttingen, 1863-1933, 1836.

Gustafsson, G., W. Baumjohann, and I. Iversen, Multi-method observations and modeling of the three-dimensional currents associates with a very strong Ps 6 event, J. Geophys., 49, 138-145, 1981.

Haines, G. V., Spherical cap harmonic analysis of geomagnetic secular variation over Canada 1960-1983, J. Geophys. Res., 90, 12563-12574, 1985a.

Haines, G. V., Spherical cap harmonic analysis, J. Geophys. Res., 90, 25832591, 1985 b.

Haines, G. V., Computer programs for spherical cap harmonic analysis of potential and general fields, Comput. Geosci., 14, 413-447, 1988.

Haines, G. V., Regional magnetic field modelling: a review, J. Geomag. Geoelectr., 42, 1001-1018, 1990.

Haines, G. V. and J. M. Torta, Determination of equivalent current sources from spherical cap harmonic models of geomagnetic field variations, Geophys. J. Int., 118, 499-514, 1994.

Hobson, E. W., The Theory of Spherical and Ellipsoidal Harmonics, pp. 500, Cambridge University Press, New York, 1931.

Inhester, B., J. Untiedt, M. Segatz, and M. Kürschner, Direct determination of the local ionospheric Hall conductance distribution from twodimensional electric and magnetic field data, J. Geophys. Res., 97, 40734083, 1992.

Press, W. H., B. P. Flannery, S. A. Teukolsky, and W. T. Vetterling, Numerical Recipes, 2nd ed., pp. 973, Cambridge University Press, Cambridge, 1992.

Richmond, A. D. and W. Baumjohann, Three-dimensional analysis of magnetometer array data, J. Geophys., 54, 138-156, 1983.

Torta, J. M. and A. De Santis, On the derivation of the Earth's conductivity structure by means of spherical cap harmonic analysis, Geophys. J. Int., 127, 441-451, 1996.

Torta, J. M., A. Garcia, J. J. Curto, and A. De Santis, New representation of geomagnetic secular variation over restricted regions by means of spherical cap harmonic analysis: application to the case of Spain, Phys. Earth Planet. Inter., 74, 209-217, 1992.

Untiedt, J. and W. Baumjohann, Studies of polar current systems using the IMS Scandinavian magnetometer array, Space Sci. Rev., 63, 245-390, 1993.

Viljanen, A., K. Kauristie, and K. Pajunpää, On induction effects at EISCAT and IMAGE magnetometer stations, Geophys. J. Int., 121, 893-906, 1995.

Walker, J. K., V. Y. Semenov, and T. L. Hansen, Synoptic models of high latitude magnetic activity and equivalent ionospheric and induced currents, J. Atmos. Terr. Phys., 59, 1435-1452, 1997.

O. Amm (e-mail: Olaf.Amm@fmi.fi) and A. Viljanen 